\title{
It's Practice, with Sleep, that Makes Perfect: Implications of Sleep-Dependent Learning and Plasticity for Skill Performance
}

\author{
Matthew P. Walker, $\mathrm{PhD}^{\mathrm{a}, *}$, Robert Stickgold, $\mathrm{PhD}^{\mathrm{b}}$ \\ a Sleep and Neuroimaging, Center for Sleep and Cognition, Department of Psychiatry, \\ Beth Israel Deaconess Medical Center, Harvard Medical School, \\ 330 Brookline Avenue, Boston, MA 02215, USA \\ ${ }^{\mathrm{b}}$ Center for Sleep and Cognition, Department of Psychiatry, Harvard Medical School, \\ Beth Israel Deaconess Medical Center, 330 Brookline Avenue, Boston, MA 02215, USA
}

When contemplating the learning of new skilled actions and behaviors, particularly those involved in athletic endeavors, practice is often believed to be the only determinate of improvement. Although repeatedly performing a new task often results in learning benefits, leading to the adage "practice makes perfect," a collection of studies over the past decade has begun to change this concept. Instead, these reports suggest that after initial training, the human brain continues to learn in the absence of further practice, and that this delayed improvement develops during sleep. This article reviews these studies of what is now becoming known as sleep-dependent memory processing and focuses on the effects of sleep in the development of procedural skills.

\section{Definitions}

Before interactions between sleep and memory can be discussed, what these terms represent and encompass must first be understood. The process of sleep, with its varied stages and equally diverse physiology and biology, has already

This work was supported by grants from the National Institutes of Health (MH 48,832, MH 65,292, MH 69,935, and MH 67,754) and the National Science Foundation (BCS-0121953).

* Corresponding author.

E-mail address: mwalker@hms.harvard.edu (M.P. Walker). 
been described elsewhere in this issue. Just as sleep cannot be considered homogeneous, the spectrum of memory categories that are believed to exist in the human brain and the unique stages that create and sustain memory appear equally diverse.

\section{Memory categories}

Although it is often used as a unitary term, memory is not a single entity. Human memory has been subject to several different classification schemes. The most popular is based on the distinction between declarative and nondeclarative memory (Fig. 1A) [1,2]. Declarative memory refers to consciously accessible memories of fact-based information (ie, knowing "what"). Several subcategories of the declarative system exist, including episodic memory (ie, memory for events of one's past) and semantic memory (ie, memory for general knowledge, not tied to a specific event) [1]. In contrast, nondeclarative memory can be regarded as nonconscious. The nondeclarative category includes procedural memory (ie, knowing "how"), such as the learning of actions and complex skills, and nonconscious implicit learning. However, although these

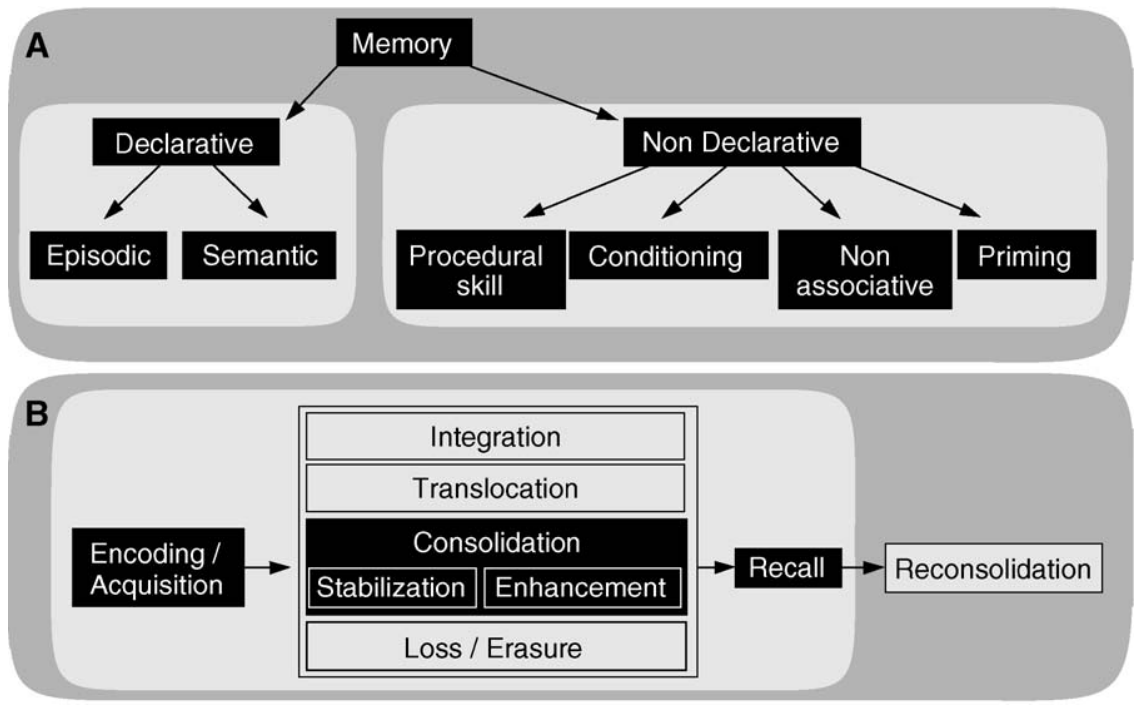

Fig. 1. Memory systems and memory stages. $(A)$ Memory systems. Human memory is most commonly divided into declarative forms, subdivided into episodic and semantic, and nondeclarative forms, subdivided into an array of different types, including procedural memory required for learning skilled actions and movements. (B) Developing stages of memory. Following initial encoding/ acquisition of a memory, several ensuing stages are proposed, beginning with consolidation, and including integration of the memory representation, translocation of the representation, or erasure of the memory. Following later recall, the memory representation is believed to become unstable again, requiring periods of reconsolidation. 
categories offer convenient and distinct divisions, they rarely operate in complete isolation and commonly work together to achieve cognitive goals.

\section{Memory stages}

Because memory is not monolithic, there is not one individual event that creates or develops it. Instead, memory appears to evolve in several unique stages over time (Fig. 1B). Memories are initially formed or acquired by engaging with an object or performing an action, leading to the rapid formation (within seconds to minutes) of a memory representation within the brain. The most efficient time for actively encoding or acquiring memory representations occurs not in sleep, but while we are awake, during a state of focused perceptual attention to external stimuli and the conscious performance of motor output.

Following acquisition, the most commonly recognized next-stage of memory formation is consolidation. Classically, consolidation refers to a stabilizing process whereby a memory becomes increasingly resistant to interference from competing or disrupting factors over time (within hours to days) [3]. However, recent findings suggest that memory consolidation is more specifically determined by time spent in unique brain states, such as sleep and the different stages of sleep. Furthermore, these studies have begun to indicate that consolidation can result not only in stabilizing memories, but actually enhancing them across sleep [4]. Although this article focuses on consolidation and the enhancing effects that develop across sleep periods, several additional memory stages beyond consolidation are also proposed, although they are currently not as well understood (Fig. 1B).

To summarize, human memory can be classified into a variety of forms, including procedural skill memory. Furthermore, the development of efficient and improved memory representations does not occur in a single step, but instead progresses through a number of distinct stages that appear to depend on the passage of time in specific brain states, such as sleep and its different stages.

\section{Behavioral studies of sleep and memory}

Sleep-dependent memory consolidation has been found in numerous species and across a range of memory systems. This article focuses on nondeclarative procedural skill memory in humans, spanning motor and sensory perceptual systems (eg, visual, auditory).

\section{Motor learning and overnight sleep}

Motor skills have been broadly classified into two forms: motor adaptation (eg, learning to use a computer mouse) and motor sequence learning (eg, learning a piano scale) [5]. However, real-life skilled procedures often involve combined use of these systems. An excellent illustration would be a pommel 
horse gymnastic routine. Not only is there a critical need to execute a series of hand and leg movements in a temporally correct sequential order (motor sequence learning) but these movements must adapt to each limb's speed and rotation at any given moment in time, compensating for the weight, velocity, and gravitational forces with which they are moving (motor adaptation learning). Clearly, these different forms of motor skill ability are equally essential in athletic performance, and therefore evidence describing sleep-dependent memory enhancement across these different motor skill forms should be considered.

Beginning with motor sequence learning, the authors have demonstrated that a night of sleep can trigger significant performance improvements in speed and accuracy of a sequential finger-tapping task [6]. In the first of a series of studies, subjects trained on the motor sequence task either at $10 \mathrm{AM}$ or $10 \mathrm{PM}$ and then retested at subsequent intervals across 24 hours. Initial practice on the motor skill task significantly improved performance within the training session for all groups equally, regardless of time of day. However, subjects then demonstrated remarkably different time courses of subsequent motor skill improvement, specifically dependent on sleep. Subjects who were trained at 10 AM in the morning showed no significant improvement when retested later that same day after 12 hours of wake time (Fig. 2A). However, when retested a second time the next morning following a night of sleep, they averaged a $20 \%$ improvement in speed and a 39\% improvement in accuracy. In contrast, subjects trained at $10 \mathrm{PM}$ in the evening immediately demonstrated equally large improvements the next morning following sleep, yet showed no significant additional improvement after another 12 hours of wake time later that day (Fig. 2B). Thus,

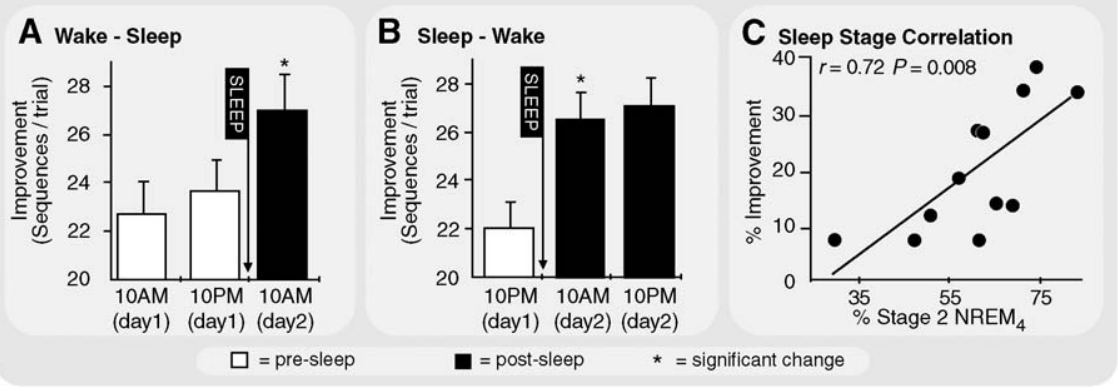

Fig. 2. Sleep-dependent motor skill learning in the human brain. $(A)$ Wake-sleep. After morning training (10 AM, clear bar) subjects showed no significant change in performance when tested after 12 hours of wake time (10 PM, clear bar). However, when tested again following a night of sleep (10 AM, filled bar), performance had improved significantly. (B) Sleep-wake. After evening training (10 PM, clear bar), subjects displayed significant performance improvements just 12 hours after training following a night of sleep (10 AM, filled bar), yet expressed no further significant change in performance following an additional 12 hours of wake time (10 PM, filled bar). (C) The amount of overnight improvement on the motor skill task correlated with the percentage of stage-2 NREM sleep in the last (fourth) quarter of the night (stage-2 NREM 4 ). Asterisks indicate significant improvement relative to training and error bars indicate SEM. 
significant delayed learning without further practice was only seen across a night of sleep and not over an equivalent period of time awake, regardless of whether the time awake or time asleep came first.

An alternative explanation of these results, however, is that motor activity during the daytime interfered with memory consolidation, and thus sleep is simply a passive time of hand rest that allows for memory enhancement. To eliminate this possibility, an additional group of subjects were trained at $10 \mathrm{AM}$, and then wore mittens for the duration of the waking interval to prevent skilled finger movements and the potential for interference before being retesting at $10 \mathrm{PM}$. Once again, the waking episode, even with total hand rest, conferred no significant improvement in performance, and actually led to a decrease in accuracy. However, when subjects returned following a night of sleep, large improvements in speed and accuracy were expressed again at retesting. Finally, when the degree of overnight learning was correlated with sleep-stage recordings, a significant positive correlation with the percentage of stage- 2 non-rapid eye movement (NREM) sleep was evident, particularly in the last quarter of the night (eg, the last 2 hours of an 8-hour night of sleep) (Fig. 2C). This window corresponds to a time when sleep spindles, which are a defining electrophysiologic characteristic of stage-2 NREM, reach peak density late in the night [7]. Spindles may trigger key intracellular mechanisms required for synaptic plasticity [8], and are known to increase following training on a motor skill task [9]. As such, this late-night sleep, laden with spindles, may offer an ideal environment for triggering events that result in plastic brain changes leading to improved skill performance. Furthermore, this late-night correlation is of particular interest when considering optimal skill learning in athletes, suggesting that early morning awakenings may prevent maximization of learning potential by cutting short a critical sleep-consolidation time window.

Fischer et al [10] confirmed these findings of sleep-dependent motor-sequence learning using a similar task, although they reported a correlation with rapid eye movement (REM) sleep. They also demonstrated that if subjects were deprived of sleep the first night after training, and then allowed a night of recovery sleep before being retested, normal overnight consolidation improvements were blocked. This finding indicates that sleep on the first night following training is critical for the development of consolidation and delayed learning. From the perspective of motor-memory consolidation, it appears that one cannot accumulate a sleep debt and hope to repay it at a later time. Instead, the sleepdependent consolidation process critically depends on sleep within the first 24 hours after training.

In another study, the authors investigated the temporal evolution of motor learning before and after sleep, the effects of different training regimens, and the long-term development of motor learning across multiple nights of sleep [11]. These data demonstrate that doubling the duration of initial training does not alter or inhibit the amount of subsequent sleep-dependent learning. Furthermore, the data show that the amount of practice-dependent learning during initial training does not correlate with the amount of subsequent sleep-dependent 
learning overnight, suggesting that these two stages (initial acquisition of the skill and the later sleep-dependent skill enhancement) are functionally distinct and regulated by different brain mechanisms. Finally, while the majority of sleep-dependent motor skill learning appears to occur during the first night of sleep, two additional nights of sleep trigger continued consolidation enhancement, with speed improving by $26 \%$ overall, and accuracy improving by nearly $50 \%$.

Because most of the skills acquired throughout life are of a multi-limb and multi-digit nature, the authors studied the effects of increasing task complexity on sleep-dependent motor learning [12]. In this study, subjects trained on a variety of task configurations involving either a short or long motor sequence, coordinated between either one or two hands. The more complex the task became (depending on the combinations of these factors), the greater the overnight, sleepdependent memory enhancement. This indicates that as task difficulty increases, the overnight sleep-dependent process responds with even greater performance improvements, further emphasizing the importance of sleep in learning many real-life motor skill routines.

These findings alone, however, do not reveal how the sleep-dependent process triggered significant improvements in motor performance speed and accuracy. Therefore, a more detailed analysis focused on performance changes within these motor sequences [12]. Before sleep, individual key-press transitions within the sequence were uneven (Fig. 3A, clear circles), with some transitions seemingly easy (fast) and others problematic (slow), as if the entire sequence was being parsed into smaller subsequences during initial learning (a phenomenon
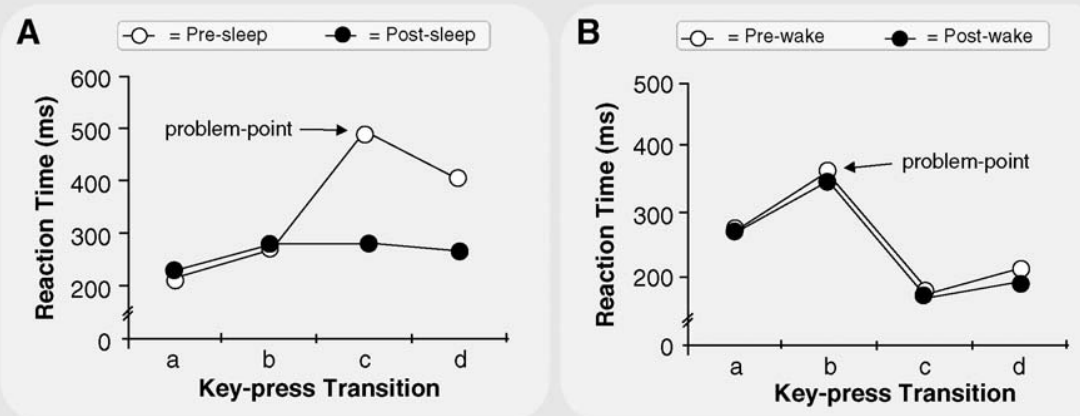

Fig. 3. Single-subject examples of changes in transition speeds. Within a five element motor sequence (eg, "4-1-3-2-4"), there are four unique key press transitions: (1) from 4 to 1, (2) from 1 to 3, (3) from 3 to 2, and (4) from 2 to 4 . (A) The transition profile at the end of training before sleep (O) demonstrated considerable variability, with certain transitions being particularly slow (most difficult; "problem points"), whereas other transitions appear to be relatively rapid (easy). Following a night of sleep (๑), there was a specific reduction (improvement) in the time required for the slowest problem point transition. $(B)$ Similarly, at the end of training before a waking interval, transition profiles were uneven (O); with some particularly slow transitions ("problem points"), and other relatively fast transitions (easy). However, in contrast to postsleep changes, no change in transition profile was observed following 8 hours of wake $(\bullet)$. 
termed chunking [13]).After a night of sleep, the problematic slow transitions improved, whereas transitions that had already been effectively mastered before sleep did not change (see Fig. 3A, filled circles). In contrast, if subjects were retested after an 8-hour waking interval across the day, the authors observed no improvement in the profile of key-press transitions at any location within the sequence (Fig. 3B). These changes suggest that the sleep-dependent consolidation process may involve the unification of smaller motor memory units into one single memory element by selectively improving problem regions of the sequence. This overnight process would therefore offer a greater degree of performance automation and effectively optimize skill speed throughout the entire motor program.

A recent study by Robertson et al [14] indicates that awareness of what is being learned can modify the sleep-dependent nature of delayed learning on a visuomotor sequence task. If subjects learn a motor sequence implicitly, that is, without conscious awareness because the sequence length exceeds the limits of explicit knowledge (eg, a particularly long 12-element motor sequence), then delayed learning improvements can develop across time awake in addition to during sleep. However, if subjects are explicitly aware of the sequence being learning, usually during shorter motor sequences, then delayed improvements again only develop across sleep, and this overnight learning correlates positively with NREM sleep. This suggests that an individual's level of awareness of what is being learned can modify the influence of wake and sleep. However, it remains unknown whether the improvements that develop implicitly across wake and sleep are achieved by similar or different underlying brain mechanisms.

Moving from motor sequence learning to motor adaptation learning, Smith and MacNeill [15] have shown that selective sleep deprivation impairs retention of a visuomotor adaptation task. All subjects trained on the task and were retested 1 week later. Some subjects were either completely or selectively deprived of different sleep stages across the first night following memory acquisition. Not all subjects retained the skill memory at the later retest session. Those subjects who were deprived of stage-2 NREM sleep expressed the most pronounced deficits in motor performance, again suggesting that stage-2 NREM is crucial to successful motor memory enhancement.

Huber et al [16] similarly demonstrated that following initial memory acquisition of a motor-reaching adaptation task, delayed learning is observed exclusively across a night of sleep, and not across equivalent periods awake. Furthermore, using high-density electroencephalography (EEG), they were able to show that daytime motor skill practice was accompanied by a discrete increase in the subsequent amount of NREM slow-wave EEG activity over the parietal cortex. They also demonstrated that this increase in slow-wave activity was proportional to the amount of delayed learning that developed overnight, and that the subjects who showed the greatest increase in slow-wave activity in the parietal cortex demonstrated the largest motor skill enhancements the next day.

In summary, these reports build a convincing argument in support of sleepdependent learning across several forms of motor skill memory. All of these 
studies indicate that a night of sleep triggers delayed learning, without the need for further training. In addition, overnight improvements consistently display a strong relationship to NREM sleep, and, in some cases, to specific NREM sleepstage windows at different times in the night.

\section{Perceptual learning and overnight sleep}

Often skilled performance is believed to involve only movement actions such as those described earlier. However, almost all of these motor actions are guided by other sensory perceptual systems, such as vision, hearing, and touch. Therefore, these systems also represent memory domains that play a significant role in skill perfection. Similar to motor skill studies, investigations of perceptual skill learning in the visual and auditory systems have offered support for the critical role of sleep in memory consolidation and associated learning enhancement.

In the visual system, Karni and colleagues [17] demonstrated that learning of a visual texture discrimination task (identifying different oriented line bars) improves significantly following a night of sleep. Furthermore, they established that selective disruption of REM, but not NREM, sleep results in a loss of these performance gains. Gais et al [18] selectively deprived subjects of early sleep, which is normally dominated by NREM slow-wave sleep (SWS), or late-night sleep, which is normally dominated by REM and stage-2 NREM, and concluded that consolidation enhancements on this task were initiated by NREM SWSrelated processes, whereas REM sleep promotes additional enhancement.

Using the same task, the authors have shown that these enhancements are specifically sleep- and not time-dependent, similar to the characteristics of motor skill learning [19]. Furthermore, overnight learning correlated positively with the amount of early-night SWS and late-night REM sleep, proposing a two-step process requiring the sequential contribution of these different sleep stages. In addition, it was established that obtaining less than 6 hours of sleep the night following training results in no significant overnight learning, and that total firstnight sleep deprivation, even when followed by two subsequent recovery nights of sleep, blocks normal sleep-dependent improvement [20]. Again these data not only highlight the importance of sleep in the evolution of delayed learning but also indicate that adequate sleep within the first 24 hours following memory acquisition is a requirement for subsequent sleep-dependent learning enhancement.

More recent studies have begun to explore sleep-dependent auditory skill learning. Gaab et al [21] used a pitch memory task to show that regardless of whether subjects train in the morning or evening, delayed performance improvements develop only across a subsequent night of sleep and not across similar waking time periods, regardless of whether the sleep episode comes first. Atienza and colleagues [22,23] have also described evidence of time- and sleep-dependent auditory memory consolidation, together with sleep-dependent changes in brain-evoked response potentials. Although posttraining sleep deprivation did not prevent continued improvements in behavioral performance, changes in evoked responses that are normally associated with the automatic 
shift of attention to relevant stimuli failed to develop following a night of posttraining sleep deprivation. Finally, Fenn et al [24] have shown that periods of wake following training on a synthetic speech recognition task actually result in deterioration of task performance across the day; an impairment that was not simply a consequence of circadian test time. However, if subjects were retested following a subsequent night of sleep, performance was restored to posttraining levels, reflecting a process of sleep-dependent consolidation capable of rescuing and reestablishing previously learned auditory skills.

In summary, learning of perceptual skills, like motor skills, appears to depend on sleep for the development of delayed learning, with evidence that several different sleep stages may be involved in triggering this form of overnight consolidation.

\section{Daytime naps and skill learning}

Although the majority of sleep-dependent studies have investigated learning across a night of sleep, several reports have examined the benefits of daytime naps on perceptual and motor skill tasks.

Based on evidence that motor learning continues to develop overnight, the authors explored the influence of daytime naps using the sequential fingertapping task [25]. Two groups of subjects trained on the task in the morning. One group then obtained a 60- to 90-minute midday nap while the other group remained awake. When retested later that same day, subjects who experienced a 60 - to 90-minute nap displayed a significant learning enhancement of nearly $16 \%$, whereas subjects who did not nap failed to show any significant improvement in performance speed across the day (Fig. 4). However, when subjects were retested a second time following a subsequent full night of sleep, those subjects in the nap group showed only an additional $7 \%$ overnight increase in speed, whereas subjects in the control group, who had not napped the previous day, displayed speed enhancements of nearly $24 \%$ following the night of sleep (see Fig. 4).

These results demonstrate that as little as 60 to 90 minutes of midday sleep is sufficient to produce significant improvements in motor skill performance, whereas equivalent periods of wake time produce no enhancement. These data also suggest that there may be a limit to how much sleep-dependent motor skill improvement can occur over 24 hours, and that napping changes the time course of when learning occurs but not how much total delayed learning ultimately accrues. Thus, although both groups improved by approximately the same total amount 24 hours later (see Fig. 4), the temporal evolution of this enhancement was modified by a daytime nap. These findings offer the exciting possibility that naps may protect motor skill memories against the detrimental effects of subsequent sleep deprivation [10], suggesting a prophylactic strategy against learning deficits if overnight sleep loss can be predicted in advance.

As with motor skill learning, daytime naps also appear to benefit visual skill learning, although the characteristics of these effects are subtly different. Med- 


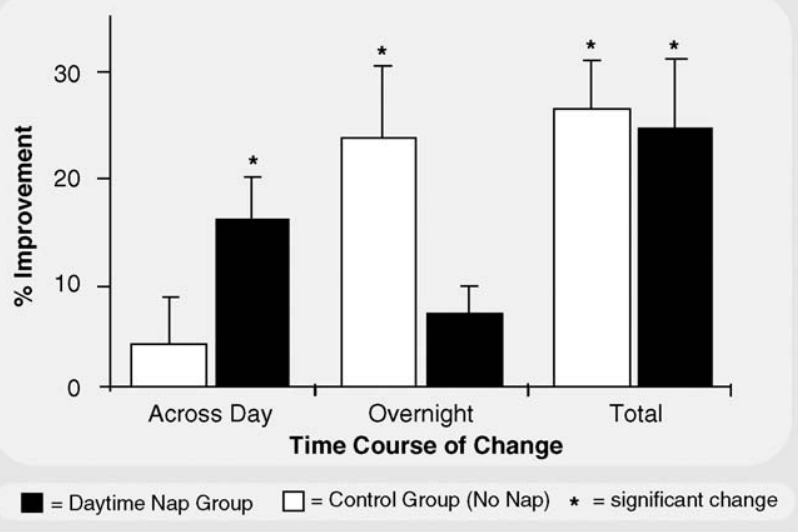

Fig. 4. Daytime naps and motor skill learning. Subjects practiced the motor skill task in the morning, and either obtained a 60- to 90-minute midday nap, or remained awake across the first day. When retested later that same day, subjects who experienced a 60- to 90-minute nap (filled bar; "Across Day") displayed significant performance speed improvements of $16 \%$, whereas subjects who did not nap showed no significant enhancements (clear bars; "Across Day"). When retested a second time after a full night of sleep the next day, subjects in the nap group showed only an additional 7\% increase in speed overnight ( filled bar; "Overnight"), whereas subjects in the control group expressed a significant $24 \%$ overnight improvement following sleep (clear bar; "Overnight"). Therefore, 24 hours later, both groups averaged the same total amount of delayed learning (filled \& clear bars; "Total"). Asterisks indicate significant improvement and error bars indicate SEM.

nick and colleagues [26] have shown that if a visual skill task is repeatedly administered across the day, performance does not improve or remain stable, but deteriorates. This may reflect a selective fatigue of brain regions recruited during task performance, a characteristic not observed in the motor system. However, if a short 30 to 60 minute daytime nap is introduced during these repeat administrations of the visual skill task, the performance deterioration is ameliorated. If a longer nap period is introduced that ranges from 60 to 90 minutes and contains REM sleep and NREM SWS, performance not only returns to baseline, but is enhanced [27]. Furthermore, these benefits did not prevent additional significant improvements across the following night of sleep, in contrast to findings reported earlier for a motor skill task.

These studies build a cohesive argument that daytime naps confer a robust learning benefit to visual and motor skills, and in the case of visual skill learning, are capable of restoring performance deterioration caused by repeated practice across the day. From the standpoint of athletic training regimens, it will be important to determine the optimal nap duration to generate maximal learning improvements and identify whether naps at different circadian times across the day (resulting in different sleep-stage compositions and electrophysiologic characteristics) are more or less effective at enhancing different forms of procedural skills. For example, delayed motor learning that develops across just a 
60 to 90 minute nap (almost equivalent to a night of sleep) may be related to the distribution and incidence of sleep spindles at this time of day. Moreover, the efficacy of shorter naps (10-20 minutes) in triggering delayed skill learning, such as those often used when one cannot pay the price of sleep inertia (discussed by Lack et al elsewhere in this issue), have not yet been evaluated. Determining optimal nap duration represents an important future research goal, considering that immediate performance after awakening from longer sleep bouts (>60 minutes) can result in short-term performance impairments caused by transient sleep inertia, potentially masking sleep-dependent improvements.

\section{Intermediate summary}

Studies in humans demonstrate that sleep is necessary for consolidation and delayed-learning enhancement across a range of human procedural skills; is able to restore previously deteriorated task performance; and can trigger additional learning improvements without the need for added practice. Furthermore, different memory systems appear to require subtly different sleep stages, or even sleep-stage time windows, for consolidation and overnight improvement.

\section{Sleep-dependent brain plasticity}

Memory formation depends on a process termed brain plasticity, which is a lasting structural or functional change in the neural response to a stimulus, such as an experience. Evidence of sleep-dependent plasticity would greatly strengthen the claim that sleep is a critical mediator of memory consolidation. In the following section, the authors consider data describing sleep-dependent brain plasticity in humans, complementing the effects of sleep on behavioral performance.

\section{Modification of post-training sleep and brain activation}

Several studies have investigated whether initial daytime training is capable of modifying functional brain activation during later sleep episodes. Based on earlier studies involving animals, neuroimaging experiments have explored whether the signature pattern of brain activity elicited while practicing a memory task actually reemerges or is replayed during sleep.

Using brain imaging, Maquet and colleagues [28] have shown that patterns of brain activity expressed during motor skill training reappear during subsequent REM sleep, whereas no change in REM sleep brain activity occurred in subjects who received no daytime training (Fig. 5). Furthermore, these researchers demonstrated that the extent of learning during daytime practice exhibits a positive relationship to the amount of reactivation during REM sleep [29]. These find- 


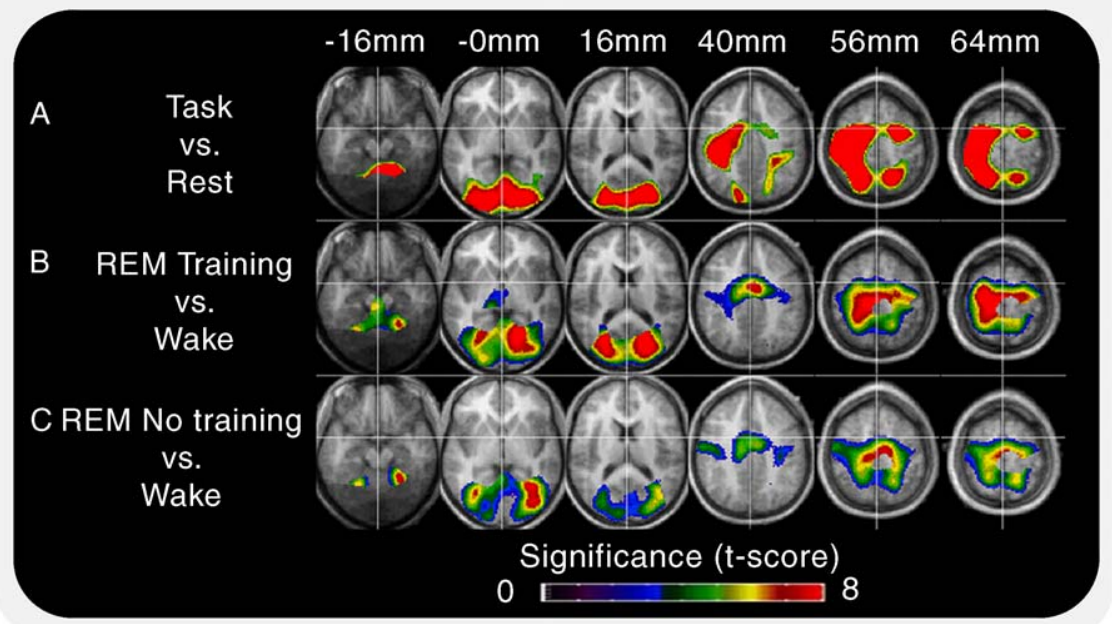

Fig. 5. Task-dependent reactivation of human brain activity during REM sleep. Statistical activation maps of different experimental contrasts. Maps are displayed at six different brain levels (from $16 \mathrm{~mm}$ below to $64 \mathrm{~mm}$ above the bicommissural plane), superimposed on the average MRI image of subjects. All maps are thresholded at $P<.001$ (uncorrected), except for $(A)$, which is thresholded at voxel-level-corrected $P<.05$. ( $A$ ) Brain regions activated during daytime performance of the motor skill task (Task versus Rest). $(B)$ Brain regions activated during subsequent REM sleep in subjects that received daytime training (REM Sleep Training versus Wake). Note considerable overlap with daytime task-dependent activity patterns. $(C)$ Brain regions activated during REM sleep in subjects who did not receive any daytime training (REM Sleep No Training versus Wake). (From Maquet P, Laureys S, Peigneux P, et al. Experience-dependent changes in cerebral activation during human REM sleep. Nat Neurosci 2000;3(8):831-6; with permission.)

ings suggest that simply experiencing the task is not what modifies subsequent sleep physiology, it is the process of learning itself. The function of sleepdependent replay may be to modify the strength of synaptic connections between neurons within specific brain networks, strengthening some synaptic connections while weakening others in the endeavor of refining skill memory.

\section{Overnight reorganization of memory representations}

An alternative approach to investigating sleep-dependent plasticity is to compare patterns of brain activation before and after a night of sleep. In contrast to measuring changes in functional activity during sleep, this approach aims to find evidence that the neural representation of a memory has been reorganized following a night of sleep.

Although the behavioral characteristics of sleep-dependent motor-sequence learning are now well established, the neural basis of this overnight learning is still unknown. Using a motor-sequence task, the authors recently explored differences between patterns of brain activation before and after sleep using func- 
tional magnetic resonance imaging (fMRI) [30]. Following a night of sleep, relative to an equivalent intervening period awake, the authors identified increased postsleep activation in motor control structures of the right primary motor cortex (Fig. 6A) and left cerebellum (Fig. 6B) — changes that likely allow faster motor output and more precise mapping of key-press movements. There were also regions of increased activation in the medial prefrontal lobe and hippocampus (Figs. 6C and 6D), structures that support improved sequencing of motor movements in the correct order. In contrast, the authors identified decreased activity postsleep bilaterally in the parietal cortices (Fig. 6E), possibly reflecting a reduced need for conscious spatial monitoring, and regions of signal decrease throughout the limbic system (Figs. 6F and 6G), indicating a decreased emotional task burden. These results suggest that sleep-dependent motor learning is associated with a large-scale plastic reorganization of memory throughout several brain regions, allowing skilled motor movements to be executed more quickly, accurately, and automatically following sleep. Furthermore, these findings hold important implications for understanding the brain-basis for perfecting real-life skills, and may also signify a potential role for sleep in clinical rehabilitation following brain damage.

Maquet et al [31] also demonstrated sleep-dependent plasticity using a combined visuomotor adaptation task. Subjects trained on the task and then were retested 3 days later, with half the subjects deprived of sleep the first night following training. The remaining half, who slept all 3 nights, showed enhanced behavioral performance at retest and a selective increase in activation in the superior temporal sulcus, a region involved in the evaluation of complex motion patterns. In contrast, subjects deprived of sleep the first night showed no such enhancement of either performance or brain activity, indicating that sleep deprivation had interfered with a latent process of neural plasticity and consolidation.

Using the sleep-dependent visual texture discrimination task described previously, Schwartz et al [32] compared performance-related fMRI patterns of brain activity 24 hours after training, relative to a naive, untrained condition. They observed significantly greater activation in the 24-hour posttraining condition in the primary visual cortex, suggesting that this functional area representing the visual memory had expended. However, these findings did not distinguish between changes that occurred during training, across posttraining wake time, or across a subsequent night of sleep.

Extending these findings, the authors examined regional brain activity patterns before and after sleep, following equivalent amounts of initial practice on this visual skill task [33]. Postsleep retesting was not only associated with significantly increased activity in the primary visual cortex but in several other visual processing regions following sleep, including at the occipital-temporal junction and in the medial temporal and inferior parietal lobes (regions involved in object detection and identification). In addition, decreased activity postsleep occurred in the right temporal pole, a region involved in emotional visual processing. These findings strengthen the claim that a night of sleep reorganizes 

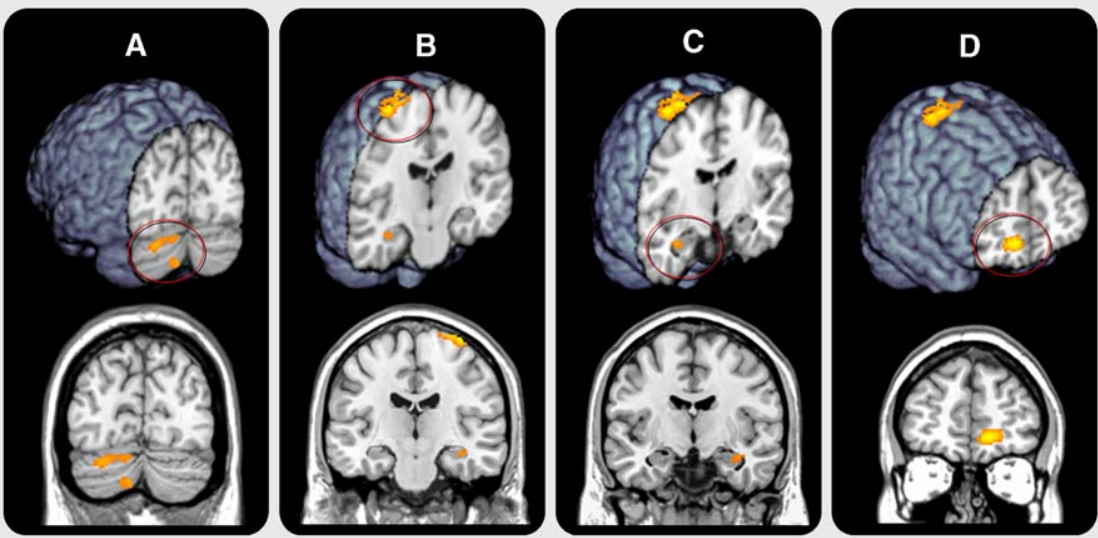

Significance (t-score)
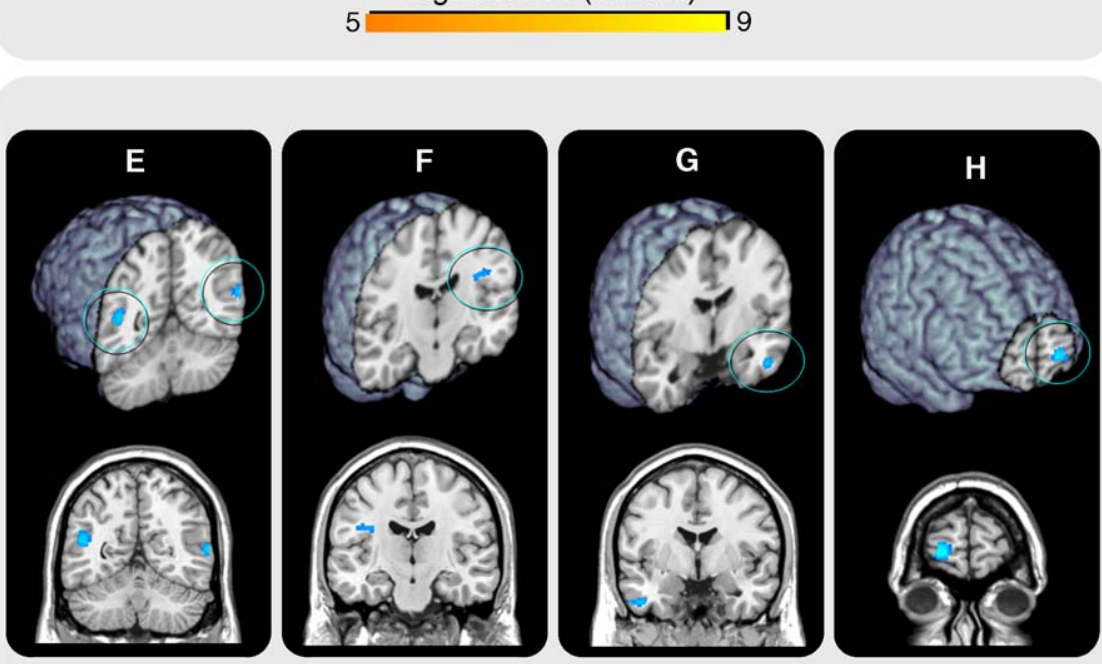

Significance (t-score)

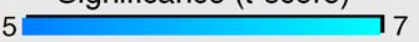

Fig. 6. Sleep-dependent brain plasticity and motor skill learning. Subjects were trained on a sleepdependent motor sequence task and retested 12 hours later, either following a night of sleep or following intervening wake, during an fMRI brain scanning session. $(A-D)$ Increased fMRI activity (red/yellow) and $(E-H)$ decreased signal activity (blue) postsleep, relative to postwake. Activation patterns are displayed on three-dimensional rendered brains (top panel of each graphic), together with corresponding coronal sections (bottom panel of each graphic). Following sleep, regions of increased activation were identified in the right primary motor cortex $(A)$, the left cerebellum $(B)$, the right hippocampus $(C)$, and the right medial prefrontal cortex $(D)$. Regions of decreased activity postsleep were expressed bilaterally in the parietal lobes $(E)$, together with the left insula cortex $(F)$, left temporal pole $(G)$, and left fronto-polar area $(H)$, all regions of the extended limbic system. All data are displayed at a corrected threshold of $P<.05$. 
the representation of a visual skill memory, with greater activation throughout the visual system following sleep perhaps offering improved identification of the visual stimulus form and its location in space.

\section{Intermediate summary}

Learning and memory are dependent on processes of brain plasticity, and sleep-dependent learning and memory consolidation must be mediated by such processes. Using brain-imaging techniques, several studies have now identified changes in the functional patterns of brain activity during posttraining sleep periods, and the reorganization of newly formed skill memories following a night of sleep. These plastic brain changes likely contribute to the refinement of the memory representation, resulting in improved next-day behavioral performance.

\section{Summary and implications}

Although the functions of the sleeping brain remain uncertain, rapidly increasing literature now supports the role of sleep in modifying and improving memory. These reports provide an abundance of converging evidence indicating that sleep-dependent mechanisms of neural plasticity lead to skill memory consolidation and consequently to delayed performance improvements. Different forms of simple and complex skill memory appear to require subtly different types of sleep for overnight memory enhancement, and several studies indicate that sleep within the first 24 hours following initial practice is essential for consolidation to develop. Furthermore, growing evidence indicates that naps can also confer a beneficial effect on skill memory, offering protection from performance deterioration and triggering memory enhancements similar to those observed across a normal night of sleep. Most recently, these findings of sleepdependent learning have been complimented by evidence of sleep-dependent plasticity within the human brain, offering a brain-based explanation for these overnight performance enhancements.

Key future research goals will be to (1) further characterize the range of sensory and motor skills that are dependent on sleep for delayed learning, (2) elucidate whether leaning concurrent skill sets involved in a single complex action (eg, learning motor adaptation and motor sequence programs simultaneously, such as in a common real-life sports scenario) either facilitates or modifies the characteristics of sleep-dependent learning, (3) determine optimal daytime nap and nighttime sleep durations for facilitating skill perfection, and how these may vary for different skill tasks, and (4) identify how much initial daytime practice should occur before an intervening sleep period is inserted, thereby developing the most optimal training routine to allow the fastest learning curve possible. 
The implications for learning skilled movements and actions are considerable, and nowhere is this more important than in the learning of procedural memory required for athletic sports. It is not uncommon to train athletes consistently across the day, only to rouse them early the next morning for the purpose of further practice. Based on current evidence, however, such a regime would appear detrimental, and by curtailing sleep durations, one runs the risk of shortchanging the brain of sleep-dependent consolidation and plasticity. Instead, building sufficient sleep periods, or even daytime sleep epochs, into training programs may offer the biologically necessary periods of sleep required to maximize skill potential, advancing learning beyond that achieved during initial practice without the need for further task engagement. If efficient skill learning is the goal, long-held beliefs may need to be modified, and the realization made that it is practice, with sleep, that ultimately leads to perfection.

\section{Acknowledgments}

We wish to thank Mysha Mason, who conducted the motor napping study reported in this manuscript.

\section{References}

[1] Tulving E. How many memory systems are there? Am Psychol 1985;40:385-98.

[2] Squire LR, Zola SM. Structure and function of declarative and nondeclarative memory systems. Proc Natl Acad Sci USA 1996;93(24):13515-22.

[3] McGaugh JL. Memory-a century of consolidation. Science 2000;287(5451):248-51.

[4] Walker MP. A refined model of sleep and the time course of memory formation. Behav Brain Sci 2005;28(1).

[5] Doyon J, Penhune V, Ungerleider LG. Distinct contribution of the cortico-striatal and corticocerebellar systems to motor skill learning. Neuropsychologia 2003;41(3):252-62.

[6] Walker MP, Brakefield T, Morgan A, et al. Practice with sleep makes perfect: sleep dependent motor skill learning. Neuron 2002;35(1):205-11.

[7] De Gennaro L, Ferrara M, Bertini M. Topographical distribution of spindles: variations between and within NREM sleep cycles. Sleep Res Online 2000;3(4):155-60.

[8] Sejnowski TJ, Destexhe A. Why do we sleep? Brain Res 2000;886(1-2):208-23.

[9] Fogel S, Jacob J, Smith C. Increased sleep spindle activity following simple motor procedural learning in humans. Paper presented at: Congress Physiological Basis for Sleep Medicine. Uruguay, October 21-26, 2001.

[10] Fischer S, Hallschmid M, Elsner AL, et al. Sleep forms memory for finger skills. Proc Natl Acad Sci USA 2002;99(18):11987-91.

[11] Walker MP, Brakefield T, Seidman J, et al. Sleep and the time course of motor skill learning. Learn Mem 2003;10(4):275-84.

[12] Kuriyama K, Stickgold R, Walker MP. Sleep-dependent learning and motor skill complexity. Learn Mem 2004;11:705-13.

[13] Sakai K, Kitaguchi K, Hikosaka O. Chunking during human visuomotor sequence learning. Exp Brain Res 2003;152(2):229-42.

[14] Robertson EM, Pascual-Leone A, Press DZ. Awareness modifies the skill-learning benefits of sleep. Curr Biol 2004;14(3):208-12. 
[15] Smith C, MacNeill C. Impaired motor memory for a pursuit rotor task following Stage 2 sleep loss in college students. J Sleep Res 1994;3(4):206-13.

[16] Huber R, Ghilardi MF, Massimini M, et al. Local sleep and learning. Nature 2004;430(6995): $78-81$.

[17] Karni A, Tanne D, Rubenstein BS, et al. Dependence on REM sleep of overnight improvement of a perceptual skill. Science 1994;265(5172):679-82.

[18] Gais S, Plihal W, Wagner U, et al. Early sleep triggers memory for early visual discrimination skills. Nat Neurosci 2000;3(12):1335-9.

[19] Stickgold R, Whidbee D, Schirmer B, et al. Visual discrimination task improvement: a multi-step process occurring during sleep. J Cogn Neurosci 2000;12(2):246-54.

[20] Stickgold R, James L, Hobson JA. Visual discrimination learning requires sleep after training. Nat Neurosci 2000;3(12):1237-8.

[21] Gaab N, Paetzold M, Becker M, et al. The influence of sleep on auditory learning: a behavioral study. Neuroreport 2004;15(4):731-4.

[22] Atienza M, Cantero JL, Dominguez-Marin E. The time course of neural changes underlying auditory perceptual learning. Learn Mem 2002;9(3):138-50.

[23] Atienza M, Cantero JL, Stickgold R. Posttraining sleep enhances automaticity in perceptual discrimination. J Cogn Neurosci 2004;16(1):53-64.

[24] Fenn KM, Nusbaum HC, Margoliash D. Consolidation during sleep of perceptual learning of spoken language. Nature 2003;425(6958):614-6.

[25] Mason M. The effects of naps and interference training on a sleep-dependent procedural motor skill learning task [Honor's Thesis]. Cambridge, Massachusetts: Harvard University; 2004.

[26] Mednick SC, Nakayama K, Cantero JL, et al. The restorative effect of naps on perceptual deterioration. Nat Neurosci 2002;5(7):677-81.

[27] Mednick S, Nakayama K, Stickgold R. Sleep-dependent learning: a nap is as good as a night. Nat Neurosci 2003;6(7):697-8.

[28] Maquet P, Laureys S, Peigneux P, et al. Experience-dependent changes in cerebral activation during human REM sleep. Nat Neurosci 2000;3(8):831-6.

[29] Peigneux P, Laureys S, Fuchs S, et al. Learned material content and acquisition level modulate cerebral reactivation during posttraining rapid-eye-movements sleep. Neuroimage 2003; 20(1):125-34.

[30] Walker MP, Stickgold R, Alsop D, et al. Sleep-dependent plasticity and motor skill learning in the human brain. Sleep 2004;27(Abstract Suppl):A405.

[31] Maquet P, Schwartz S, Passingham R, et al. Sleep-related consolidation of a visuomotor skill: brain mechanisms as assessed by functional magnetic resonance imaging. J Neurosci 2003; 23(4): $1432-40$.

[32] Schwartz S, Maquet P, Frith C. Neural correlates of perceptual learning: a functional MRI study of visual texture discrimination. Proc Natl Acad Sci USA 2002;99(26):17137-42.

[33] Walker MP, Yoo SS, Stickgold R. The functional anatomy of sleep-dependent visual skill learning. Sleep 2004;27(Abstract Suppl):A406. 